interact primarily with other fluid molecules. Relatively few are in contact with the surface of the container. At nanometer scales, however, a much higher proportion of molecules come in contact with the confining material. This surface interaction can significantly alter fluid properties, including molecular mobility, according to the research team.

As reported in the May 5 issue of Physical Review Letters (\#177804; DOI: 10.1103/PhysRevLett.96.177804), the group performed molecular dynamics simulations to study the behavior of hard-sphere fluids in highly restrictive channels with different shapes and boundary interactions. They modeled changes to fluid mobility and entropy in these conditions, a critical breakthrough that will allow engineers to learn how these changes occur while avoiding the difficult task of gathering experimental data on such small scales (see Figure 1).

"One way to think about how mobility relates to entropy is to think of entropy as measuring a sort of randomness at the molecular level," Truskett said. "In a gas, where the molecules are randomly distributed, entropy is high and the gas mixes readily. In a solid, the molecules are aligned in a regular spatial pattern; there is little randomness and the solid barely mixes at all. Our discovery is that while both excess entropy and mobility of a fluid are affected by confinement, the relationship between the two quantities essentially remains the same down to very small scales."

Because scientists already have reliable methods for predicting how confinement will affect excess entropy, they can now use this information together with the group's findings to predict how confinement will affect fluid mobility.

\section{Buried Interfaces Imaged Using Noncontact Picosecond Acoustic Microscopy}

Early identification of failures in semiconductor and nanoelectronic devices, such as delaminations and voids, is crucial so that defective parts can be screened out. Nondestructive characterization and identification of such failures is a major challenge. Scanning acoustic microscopy with a single spherical lens is typically used for inspecting such failures. The technique involves the generation of ultrasound by a piezoelectric transducer focused with a spherical lens, with both the lens and sample immersed in a coupling medium, usually water. One of the limitations of this technique is the attenuation of the ultrasound at high frequencies. In addition, immersion in the coupling medium ren- ders samples unsuitable for additional processing. To overcome these shortcomings, a new noncontact, nondestructive technique that involves generating and detecting ultrahigh-frequency acoustic waves directly on the sample has now been reported. S. Ramanathan (formerly at Intel Corp. and now at Harvard University) and D. Cahill of the University of Illinois at Urbana-Champaign describe this technique in the May 2006 issue of the Journal of Materials Research (p. 1204; DOI: 10.1557/JMR.2006.0141).

The technique involves coating the sample with an 80-nm-thick aluminum film that acts as a transducer to generate and detect acoustic pulses. In this case, the sample consisted of a silicon wafer covered by an oxide layer, an etch-stop layer, and a second oxide layer in which an array of copper metal lines was fabricated. The copper lines were each $6 \mu \mathrm{m}$ wide, $40 \mu \mathrm{m}$ long, and $1 \mu \mathrm{m}$ thick. The lines on one of these wafers were bonded to the lines on a second wafer, which was used as a handle wafer. The topmost silicon layer was then thinned to $6 \mu \mathrm{m}$, and an aluminum transducer film was deposited. A Ti:sapphire laser beam was directed onto the aluminum surface, where the laser pulse generated a local stress field, leading to a short-duration longitudinal acoustic pulse that travels from the aluminum film into

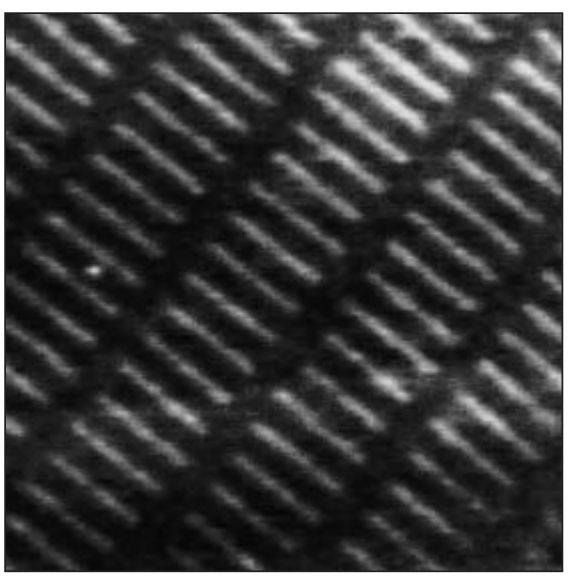

Figure 1. Acoustic microscopy image of copper lines imaged at a pump-probe delay time of 1.711 ns to select the acoustic echo from the oxide/Cu interface. The gray scale corresponds to variations in the change in optical reflectivity of approximately $15 \%$. The $6 \mu \mathrm{m} \times 40 \mu \mathrm{m}$ copper lines are clearly imaged through the 6- $\mu$ m-thick silicon substrate. The image shows a $200 \mu \mathrm{m}$ $\times 200 \mu \mathrm{m}$ area. Reproduced with permission from the Journal of Materials Research 21 (5) (2006) p. 1204; DOI: 10.1557/JMR.2006.0141. () 2006 by the Materials Research Society. the Si substrate. A fraction of the pulse is reflected back toward the surface when it encounters a discontinuity in acoustic impedence, such as at an interface. The reflectivity of the metal film is changed slightly due to these echoes, which are measured by a time-delayed probe beam from the laser. The researchers observed echoes from the silicon/oxide interface, the oxide/etch-stop interface, the oxide/ $\mathrm{Cu}$ interface, and the oxide/air interface. The researchers imaged buried regions of the sample by raster-scanning the sample beneath the pulse and probe beams at a fixed time delay. The strength of the changes in reflectivity at each sample point was collected, and the data were analyzed as an image. Figure 1 shows an example of an acoustic image of the copper lines taken at a time delay of $1.711 \mathrm{~ns}$, corresponding to the acoustic return from the oxide/ $\mathrm{Cu}$ interface. This is a grayscale representation of the ratio of the inphase and out-of-phase signals measured by an rf lock-in amplifier. The image shows a $200 \mu \mathrm{m} \times 200 \mu \mathrm{m}$ area. No image processing, such as filtration or noise reduction, was applied. The researchers also imaged an isolated oxide/etch-stop interface, which, according to the research team, would be impossible with the conventional technique.

The results of the study demonstrate the feasibility of the method as well as its potential use as an extremely sensitive probe of the mechanical reliability of interfaces. The technique has spatial resolution on the order of few micrometers, which is limited by the laser spot size, and nanoscale depth resolution. The technique can thus potentially be used for high-resolution analysis to identify failures. The researchers said that it may be possible to use a longer wavelength laser beam that is transparent to the silicon substrate, which could allow for acoustic pulse generation targeted directly at the interconnect layers, thereby overcoming the need for any external couplant.

GOPAL RAO

\section{Gold Nanorods Optically Trapped}

Optical trapping uses forces exerted by a strongly focused laser beam to trap small objects at the center of the beam. By exploiting the plasmonic property of gold nanorods, M. Pelton and co-workers at the University of Chicago have demonstrated that the rods can be optically trapped and oriented in three dimensions for more than $15 \mathrm{~min}$. Resonances are known to enhance optical forces on particles, and the researchers use the fact that the optical gradient force on the gold nanorod is greatly enhanced when the 
trapping laser wavelength is tuned to the long-wavelength side of the longitudinal plasmon resonance. Also, since the longitudinal plasmon is excited only by light whose polarization is parallel to the long axis of the rod, a linearly polarized trapping laser aligns the long axes of the rods along the polarization direction.

As reported in the July 1 issue of Optics Letters (p. 2075), gold nanorods (48 $\mathrm{nm} \pm$ $9 \mathrm{~nm}$ long) are fabricated with aspect ratios such that the longitudinal plasmon resonance is at $800 \mathrm{~nm}$. A tunable, continuouswave Ti:sapphire laser with a water- immersion microscope objective is used for trapping the nanorods in solution. A low-average-power mode-locked laser producing ultrashort probe pulses with a wavelength of $800 \mathrm{~nm}$ has its beam focused at the same spot as the trapping laser, producing two-photon fluorescence from the trapped rods. The trapping times of the rods are measured through the time trace of the two-photon fluorescence. The motion of the rods in solution shows two distinct components, one with a $5-\mu$ s characteristic decay time and one with a $20-m s$ characteristic decay time, when the continuous-wave trapping laser is turned off. The researchers attribute the $5-\mu$ decay time to rotational diffusion (tumbling) of the nanorods about their short axes, and the 20-ms decay time to translational diffusion of the nanorods through the probe laser spot. The 5 - $\mu$ s component of the decay is absent when the rods are trapped with varying laser wavelengths (790-850 $\mathrm{nm}$ ) and laser powers (60-140 $\mathrm{mW})$. Furthermore, the translational diffusion time is lengthened by the trapping process. According to the researchers, since the $5-\mu$ s decay time is

\section{Temporal Control of Nanoparticle Assembly Detects Protease Correlated with Tumor Cells}

Although self-assembly strategies found in nature exhibit both spatial and temporal control of protein activity initiation, bio-inspired nanoassembly generally has limited applicability due to a lack of temporal control. Recently, however, researchers inspired by bioassembly initiated by the enzymatic removal of inhibitors have used peptide-polymer chemistry to show that inorganic nanoparticles can be placed in a latent state by chemical modification; nanoassembly can then be initiated by the protease enzyme matrix metalloproteinase-2 (MMP-2), which is produced by tumor-derived cells. The MMP2-triggered nanoassembly thereby enables tumor detection.

As reported in the May 5 issue of Angerwandte Chemie International Edition (p. 3161; DOI: 10.1002/anie.200600259), S.N. Bhatia of the Massachusetts Institute of Technology; A.M. Derfus of the University of California, San Diego; E. Ruoslahti of the Burnham Institute in La Jolla, Calif.; and their co-researchers modified 50-nm, dextran-coated, superparamagnetic $\mathrm{Fe}_{3} \mathrm{O}_{4}$ nanoparticles with either biotin (a small molecule) or neutravidin (a tetrameric protein). The two nanoparticle populations self-assemble in solution due to highly stable biotin-neutravidin interactions. Enzymatic control of nanoparticle assembly is effected by further modifying the nanoparticle surface with the MMP-2 peptide substrate, which also serves as an anchor for linear poly(ethylene glycol) (PEG) chains (see Figure 1).

The researchers selected a PEG chain length that would inhibit nanoparticle association but still allow MMP-2 to cleave the peptide linkers holding the PEG to the nanoparticles and thereby initiate self-assembly. The researchers also demonstrated that self-assembly amplifies the transverse relaxation (T2) of $\mathrm{Fe}_{3} \mathrm{O}_{4}$ nanoparticle solutions, which is remotely detectable with magnetic resonance imaging. (Amplification of the diffusional dephasing of the water molecule surrounding the nanoassemblies shortens T2.)

Atomic force microscopy showed that the nanoassembly sizes fall in the range of $0.5-1 \mu \mathrm{m}$, which suggests that the nanoassemblies are composed of tens to hundreds of nanoparticles. The researchers also demonstrated that measurement of amplified T2 is sensitive enough to detect protease-triggered nanoassembly across a 10 -fold variation in particle concentration-from $0.7 \mathrm{mg}$ $\mathrm{Fe} / \mathrm{kg}$ to $7 \mathrm{mg} \mathrm{Fe} / \mathrm{kg}$, a range that encompasses typical working concentrations used for tumor and lymphatic targeting in vivo.

The researchers demonstrated the utility of their approach in a complex biological specimen and showed that any existing nonspecific protein adsorption was not problematic. In addition, the nanoassembly's amplified cumulative dipole allows manipulation with imposed magnetic fields so that the assembled nanoparticles can be used to target cells. The researchers said that "in the future, this general approach may enable site-selective immobilization and enhanced image contrast in regions of tumor invasion in vivo."

STEVEN TROHALAKI

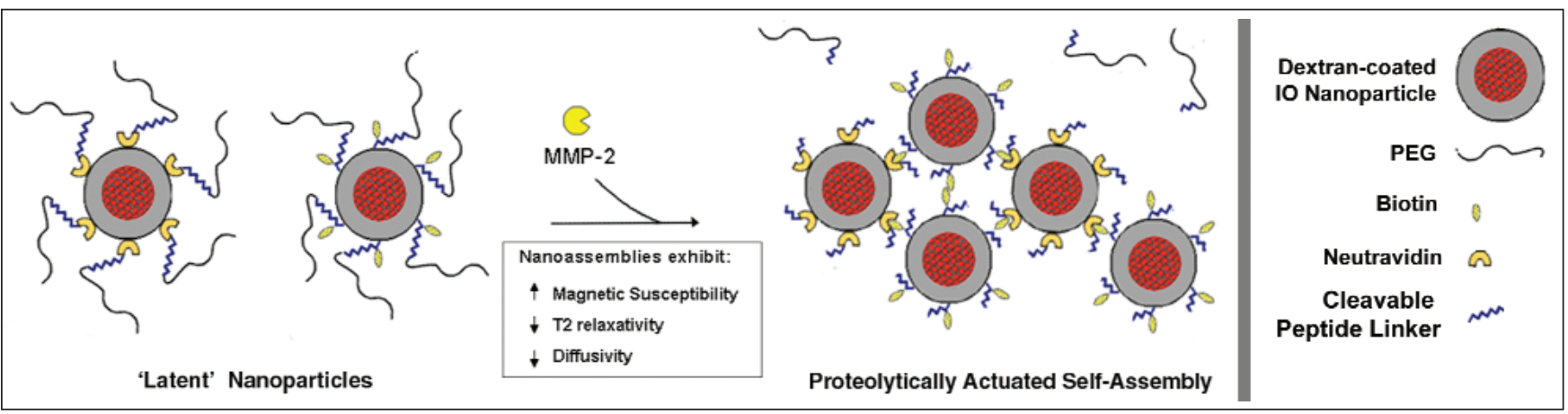

Figure 1. Schematic representation of a proteolytic actuation of self-assembly. Neutravidin- and biotin-functionalized superparamagnetic iron oxide nanoparticles are inhibited by the attachment of PEG chains that are anchored by MMP-2-cleavable peptide substrates (GPLGVRGC). Upon proteolytic removal of the PEG through cleavage of the peptides, biotin and neutravidin particles self-assemble into nanoassemblies with enhanced magnetic susceptibility, T2 magnetic-resonance relaxation, and lowered diffusivity. Reproduced with permission from Angewandte Chemie International Edition 45 (2006) p. 3161; DOI: 10.1002/anie.200600259. (c) 2006 by Wiley-VCH. 
caused by the tumbling of rods about their short axes, this result indicates that the trapping causes suppression of rotational diffusion along the short axes, so that the long axes of the rods are oriented relative to the trapping laser polarization. Also, clear signs of stably trapped rods are seen at longer times, with trapping times that follow the expected Kramer's escape rate. According to the researchers, the selectivity of optical forces to the plasmon resonance frequency could allow setting up an array of optical traps to separate metal nanoparticles of different shapes.

TUSHAR PRASAD

\section{Silicon's Role in Photonics May be Enhanced by Nanowires and Nanocones}

Silicon, the element responsible for most of the advances made in computer and electronics technology, has played a comparatively lesser role in photonic and optical devices. Although the detection of light by silicon can be very efficient, depending upon wavelength, the emission of light is very inefficient because of the indirect energy bandgap. Optical gain has been obtained from silicon only by using nanocrystals or stimulated Raman scattering. While Raman signals can be obtained from bulk crystals of silicon, the Raman scattering efficiency from a nanowire can be nearly 1000 times larger than from the bulk, depending on the diameter of the nanowire, as reported recently by L. Cao and colleagues from Drexel University.

As discussed in the April 21 issue of Physical Review Letters (\#157402; DOI: 10.1103/PhysRevLett.96.157402), graduate student Cao and professors J.E. Spanier and B. Nabet studied nanowires with widths just below and above $100 \mathrm{~nm}$, sizes which are larger than those that many researchers have been investigating. The research team also examined the effects of the variable diameter accessed when studying nanocones. The researchers prepared solid silicon nanocones (with diameters ranging from several micrometers at the base to $<5 \mathrm{~nm}$ at the tip) and silicon nanowires of selected diameters (ranging from $\sim 130 \mathrm{~nm}$ to $\sim 1 \mu \mathrm{m}$ ) by metal-catalyzed chemical vapor deposition. The researchers studied Raman scat- tering from the nanocones, nanowires, and a bulk single-crystalline $\mathrm{Si}(100)$ wafer.

Using Raman scattering spectroscopy and scanning electron microscopy (SEM) analysis, the researchers explained their experimental results in terms of strong resonant enhancement of the incident electromagnetic field inside the tiny cross section of the nanostructures when "the wavelength of the incident field is commensurate with that of an electromagnetic eigenmode of the particle, which depends upon its size and on the refractive indices of the particle and the surrounding medium." The researchers measured the Raman signal obtained from excitation at $514.5 \mathrm{~nm}\left(\mathrm{Ar}^{+}\right.$laser), $632.8 \mathrm{~nm}$ (HeNe laser), and $785 \mathrm{~nm}$ (diode laser) wavelengths. The results were consistent with their model calculations based on the dimensions of the structures observed by SEM as well as the excitation wavelengths and the polarization state of the incident light.

The researchers said that the results open the possibility of engineering silicon nanostructures to enhance the coupling of light for photonic and sensing applications.

\section{News of MRS Members/Materials Researchers}

The U.S. National Academy of Sciences has announced the election of members and foreign associates in recognition of their distinguished and continuing achievements in original research, including

Chunli Bai, Chinese Academy of Sciences;

Jillian F. Banfield, University of California, Berkeley;
Paul F. Barbara, University of Texas at Austin;

David M. Ceperley, University of

Illinois at Urbana-Champaign;

Luiz Davidovich, Federal University of Rio de Janeiro;

Mark E. Davis, California Institute of Technology;

William A. Eaton, National Institutes of Health;
Joachim Frank, New York State Department of Health;

Leslie Greengard, New York University;

Harold W. Kroto, Florida State University; and

E. Ward Plummer, University of Tennessee, Knoxville.

\section{News of MRS Corporate Affiliates/Materials Institutions}

The Alabama A\&M University Research Institute (AAMURI) MentorProtégé projects received three NunnPerry Awards at the annual Department of Defense Mentor-Protégé Conference in March. The AAMURI team consisted of SAIC, Boeing, Kemco Manufacturing, Arrowhead Contracting Inc., and Ellis Environmental Group. AAMURI, furthermore, has been selected by the Defense Information Systems Agency (DISA) as the prime contractor for the DISA Minority Institutions Technical Support Services (MITSS II) project; AAMURI will manage the $\$ 16$ million contract over the next four years with the assistance of AAMURI partners.

FEI has received four awards for the design, performance, and innovation of its $\operatorname{Titan}^{\mathrm{TM}}$ scanning transmission electron microscope (Titan S/TEM). Honors include the iF Design Award bestowed by the International Design Forum (iF) in Hanover, Germany, and the Innovative Product of the Year Award presented by the Oregon Tech Awards in the United States. The Titan S/TEM was also selected as one of the Top Products of 2005 by Solid State Technology magazine and one of the Greatest Hits of 2005 by MICRO magazine.

Flowserve Corp. has received a certificate of appreciation from the National Aeronautics and Space Administration (NASA) for custom-engineering new valves for the Johnson, Marshall, and Stennis Space Centers.

The Mitsubishi Chemical Corporation of Tokyo and the University of California, Santa Barbara (UCSB), announced in February that they are extending their research and education alliance. Under the terms of the new agreement, Mitsubishi Chemical will invest between $\$ 8.5$ million and $\$ 10$ million at UCSB over the next four years. The funds will support research as well as the administration of the Mitsubishi Chemical Center for Advanced Materials (MC-CAM) center. The total also includes a philanthropic contribution of $\$ 800,000$ to permanently endow new graduate fellowships in materials and chemical engineering.

Structured Materials Industries Inc. (SMI) has been awarded a Phase II SBIR (Small Business Innovation Research) grant by the Department of Energy to develop $p$-type doping of $\mathrm{ZnO}$. 